window seal. The fact that the authors did not find a perilymph fistula does not exclude this possibility as they can heal very rapidly, as after routine stapedectomy. I note that tinnitus and nausea had resolved by the time of surgery. Incidentally, experimental inoculation of mumps virus at the oval window does not lead to labyrinthitis in monkeys. ${ }^{3}$

A G GoRDON

London SE5

' Overman, J R, Archives of Internal Medicine, 1958, $102,354$.

Foldi, M, American Heart Yournal, 1977, 93, 121.

Karmody, C S, Annals of Otology, Rhinology and

\section{Abdominal and thoracic pressures during defecation}

SIR,-Following the article by Dr S S Fedail and others (13 January, $p$ 91) entitled "Abdominal and thoracic pressures during defecation" I would like to comment on the method and interpretation of the results.

Firstly, the technique of locating the diaphragm by the point of respiratory reversal on the manometry tracing is somewhat erroneous. The point of respiratory reversal is normally at the upper end of the highpressure zone (sphincter + diaphragm) above the lower oesophageal sphincter component. The diaphragmatic component of the highpressure zone normally appears at the lower (distal) aspect. However, the proximal tube would undoubtedly have been in the oesophagus and the pressures they measured would have accurately reflected the difference between stomach and thorax. There is naturally a significant difference between these results, but the important factor is the gastrooesophageal pressure difference. There was no significant difference between the squatters and the sitters. The authors fail to mention the important closing and antireflux mechanisms that occur at the gastro-oesophageal junction -for example, angle of implantation, mucosal flap, and right crura, all of which depend to a large extent on a positive intra-abdominal pressure.

In conclusion, I think that their idea is correct and our mode of defecation may well be a causative factor in the development of hiatal hernia. However, it is unwise to base the argument on such flimsy "not significant" evidence.

Thoracic Surgical Unit,

Papworth Hospital,

Cambridge

SIR,-Dr S S Fedail and others (13 January, p 91) put forward evidence to support the opinions of Muller ${ }^{1}$ and Burkitt and James ${ }^{2}$ that the pressure gradient between abdomen and thorax during straining at stool is an aetiological factor in hiatus hernia.

Although attractively simple, this does not withstand any detailed examination, since it fails entirely to explain why the oesophagus withdraws pari passu as the stomach advances up the mediastinum, as is the case in sliding hiatus hernia, where the oesophagogastric junction is always at the apex of the herniated stomach. The sliding type of hernia comprises the vast majority of all hiatus hernia. It fails also to explain the phenomenon of incompetence of the oesophagogastric sphincter, usually found in association with sliding hernia, but often found in its absence.

Damage to the hiatus-for example, surgical trauma-results not in the sliding type of hernia but in the paraoesophageal type, in which the fundus of the stomach, or a part of it, together with the oesophagogastric junction, are herniated into the lower mediastinum, without disturbance of function of the sphincter, and without shortening of the oesophagus. One would expect this type of hernia to result from a raised pressure gradient across the diaphragm, as in straining at stool; but it does not.

The prevalence of hiatus hernia in the West and its rarity in Africa invite the conclusion that a principal aetiological factor is diet. With this I agree; but I feel that this factor induces a disorder of oesophagogastric motility, the important feature being overactivity of the longitudinal muscle of the oesophagus. This leads to oesophageal shortening, which draws the stomach into the chest, producing the characteristic sliding hernia. It is this shortening-and thickening-which distracts, elongates, and renders incompetent the oesophagogastric sphincter. It is this shortening which often renders repair of the hernia difficult, and sometimes impossible. Surgical procedures which overcome the shortening or the tension in the oesophagus relieve the symptoms, although they need not necessarily reduce the hernia.

Unfortunately we have no means of measuring the activity of the longitudinal muscle of the oesophagus, despite having had elegant methods of measurement of the circular muscle for many years. As we cannot measure longitudinal muscle activity, we prefer to ignore it. But the longitudinal muscle is by far the most prominent feature of the oesophagus.

In short, sliding hiatus hernia is not pushed up into the chest by abdominal pressure; it is pulled up by the oesophagus. I have previously elaborated this, basing my views on surgical experience. $^{3}$

KenNeth S Mullard

Bletchington, Oxon

'Muller, C J B, South African Medical fournal, 1948, 22, 376.

Burkitt, D P, and James, P A, Lancet, 1973, 2, 128 Mullard, K S, Annals of the Royal College of Surgeon
of England, 1972, 50, 73 .

\section{Physical therapy in chronic bronchitis}

SIR,-Dr Margaret E Hodson and others (6 January, p 55) correctly observe that we did not separate the effect of intermittent positive pressure breathing (IPPB) and physiotherapy (2 December, $p$ 1525). The similar response of our treated and control groups suggests that this was an economic and wise decision, unles one considers these forms of treatment mutually antagonistic.

I would be surprised if any alteration in physiotherapy technique or method of administration of bronchodilator would produce better results than we obtained in either of our treatment groups, but shall await the evidence.

Your correspondents quoted an excellent paper by Shenfield et al as evidence that a bronchodilator is more efficient by IPPB than by aerosol. ${ }^{1}$ The actual title of the paper wa "The fate of nebulised salbutamol (Albuterol) administered by intermittent positive respiration to asthmatic patients." As the title suggests, it was concerned with the pharmacodynamics of inhaled salbutamol, and blood levels of the drug over eight hours and urine and faecal samples over three days. One could not suggest from the data presented in the paper that the use of IPPB with nebulised salbutamol was more effective than a pressurised aerosol alone, as the authors did in the summary. Furthermore, this paper referred to five convalescing asthmatics. We considered the paper by Berend et al more appropriate, as it concerned the disease we were studying and for that reason quoted it. ${ }^{2}$

The final point in the letter about the importance of physiotherapy and the use of a bronchodilator for those who have large volumes of secretions, is discussed in the Lancet editorial to which it refers. ${ }^{3}$

Service de Pneumologie,

DunCaN NewtoN Hôpital
Paris

${ }^{1}$ Shenfield, G M, et al, American Review of Respiratory Diseases, 1973, 108, 501

Berend, N, et al, British fournal of Diseases of the Chest, 1978, 72, 122.

${ }^{3}$ Newton, D A G, Lancet, 1979, 1, 49.

\section{An unsavoury custom?}

SIR,-It is one of the marks of a first-class hospital that its doctors and laboratory staff do not come to meals wearing their white coats. I know of no spread of an infection which was traced to this unsavoury custom; but why wait till something untoward occurs before discontinuing the practice where it still persists? Admittedly there are often administrative difficulties, notably in teaching hospitals where changing facilities would have to be found for numerous clinical students. The DHSS definitely frowns on this custom. If medical men do not feel concerned, perhaps the administrators in charge of hospital dining rooms and canteens ought to act and exclude clothing which might be contaminated from areas where food is served and consumed.

H LEHMANN

Department of Biochemistry, University of Cambridge

\section{Hazards of sulphinpyrazone}

SIR,-With reference to the letter on hazards of sulphinpyrazone (23-30 December, p 1786) I would like to make some comments.

Sulphinpyrazone being a pyrazole compound is related to phenylbutazone, which has been observed to potentiate the hypoglycaemic effect of the oral sulphonylureas. Hence sulphinpyrazone should be used with caution in patients on concomitant sulpha drugs, the sulphonylurea hypoglycaemic agents, and insulin.

Sulphinpyrazone is potent uricosuric agent, and alkalinisation of urine and high fluid intake are necessary at first to avoid crystalluria. In patients on phenylbutazone oedema occurs, due to a renal effect in reducing sodium and water excretion. Since sulphinpyrazone is closely related (a pyrazole) to phenylbutazone the salt retention as a side effect should also be borne in mind, for it may precipitate heart failure.

I endorse the note of caution of $\mathrm{Dr} D$ Mattingly and his colleagues that sulphinpyrazone is contraindicated in patients on oral anticoagulants. This drug is also contraindicated in patients with history of hypersensitivity 